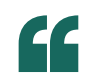

it was the

anions - the

chloride ions

- that bound

to the peptides

and were

templating

the cyclization

reaction

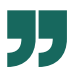

\title{
CYCLIC PEPTIDES
}

\section{Running rings around chloride}

The superior properties of cyclic peptides versus their linear counterparts in terms of bioactivity and stability have meant that they are of wide interest in drug discovery. But it is the properties of cyclic peptides as anion binders that have ultimately led to an advance in the synthesis of these important molecules. Writing in Organic Letters, Vladislav Tomišić from the University of Zagreb, Giovanna Speranza from Universita degli Studi di Milano and their co-workers have described peptide macrocylization templated by chloride ions.

Many approaches to the synthesis of cyclic peptides have been described, but the cyclization step remains the key challenge. Success inevitably relies upon some prior organization of the precursor into a conformation that brings the reactive termini of the linear peptide - amine and carboxylic acid, perhaps activated for reaction - into

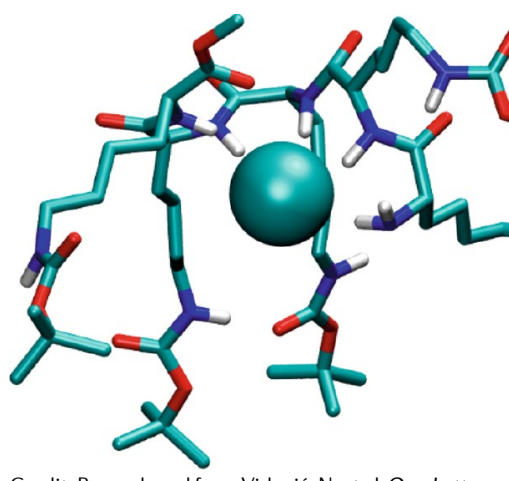

Credit: Reproduced from Vidović, N. et al. Org. Lett. 22, 2129-2134 (2020), ACS close proximity. Certain peptide sequences more readily undergo cyclization because they include turn-inducing elements, with proline residues being a popular one. More general approaches, however, tend to rely on an external template for preorganization.

Tomišić and co-workers were investigating the properties of cyclic peptides as anion receptors. "We prepared several linear peptide precursors and carried out their head-to-tail cyclization according to a literature procedure based on the metal-ion assisted ring closure," he explains. Although these reactions were successful, they afforded only poor to moderate yields of the cyclized products.

In an attempt to understand the supposed cation-assisted preorganization, the team undertook a series of NMR spectroscopic titrations and performed microcalorimetric measurements on ester protected analogues of the linear precursors. "Our results with isothermal calorimetry and NMR led us to conclude that it was the anions the chloride ions - that bound to the peptides and were templating the cyclization reaction," says Tomišić.

The most efficient cyclization reactions resulted from the use of tetraalkylammonium chlorides, presumably because the anion is more freely available as a template. When a reaction was attempted in the presence of tetraethylammonium acetate, no cyclic product could be detected. Molecular dynamics simulations provided additional information on the pre-organized structure of linear peptides in their chloride complexes.

Although initial experiments had focused on the cyclization of lysine oligomers (with tert-butoxycarbonyl protection on the side chains), which are known to be challenging to cyclize, the scope of this procedure was then explored further with a series of other peptides incorporating leucine, isoleucine, phenylalanine and serine. In all cases, the yields of cyclized product significantly exceeded the results achieved using metal templating.

There remains much to do to develop the method. "We would like to gain a more detailed insight into the roles of cations, anions and various coupling reagents and the corresponding mechanisms of ring closure," says Tomišić. "We'd particularly like to investigate the effect of oxoanions as templates since they are capable of forming stronger hydrogen bonds, which might result in more stable complexes with linear peptides and thus a more efficient templating action."

Stephen G. Davey

ORIGINAL ARTICLE Vidović, N. et al. Chlorideassisted peptide macrocyclization. Org. Lett. 22, 2129-2134 (2020) 